\title{
Prevalence of extended-spectrum betalactamase producing-Enterobacteriaceae (ESBL-E) carriage on admission at Geneva University Hospitals (HUG)
}

\author{
C Fankhauser $^{1 *}$, J Schrenzel ${ }^{2}$, V Prendki ${ }^{3}$, F Ris ${ }^{4}$, E Schiffer $^{5}$, P Gastmeier ${ }^{6}$, Y Carmeli ${ }^{7}$, D Pittet ${ }^{1}$, S Harbarth ${ }^{1}$ \\ From 3rd International Conference on Prevention and Infection Control (ICPIC 2015) \\ Geneva, Switzerland. 16-19 June 2015
}

\section{Introduction}

The increasing prevalence of ESBL-E in the community is a cause of concern for hospitals. Early detection of ESBL-E carriers on admission could allow timely implementation of control measures or appropriate selection of antimicrobials.

\section{Objectives}

To describe the current prevalence of ESBL-E rates upon admission to 4 different services at HUG, in the context of a multicenter European study (R-Gnosis).

\section{Methods}

Patients admitted to 4 different services were screened by rectal swabs on admission. From January 2014 through January 2015, patients admitted to 4 wards, including: Ortho1 (sport traumatology), Ortho 2 (septic); Geriatrics (2 wards) and patients undergoing elective colorectal surgery (ECS) were screened from April 2013-October 2014.

\section{Results}

Overall, from 2394 admitted patients, 2136 were screened on admission (89.2\%). Median age was 67.3 years (SD $\pm 20.9)$; $51.6 \%$ were male. Only $92 / 2136(4.3 \%)$ had a previously known status of ESBL carriage. A total of 226/2136 (10.6\%) patients were found to be ESBL-E carriers: $E$. coli $(\mathrm{n}=166 ; 73.4 \%) ;$ K. pneumoniae $(\mathrm{n}=26 ; 11.5 \%)$ and other Enterobacteriaceae ( $\mathrm{n}=34 ; 15.0 \%)$. Among K. pneumonia carriers on admission, 24/26 (92.3\%), had a previous hospitalization less than 12 months before admission screening and 21/26 (80.8\%) had the previous hospitalization within 3 months only. ESBL-E carriage was 83/981 (8.4\%) and 61/430 (14.2\%) for Ortho 1 and 2 respectively; Geriatrics, 42/371 (11.3\%); and ECS, 41/354 (11.6\%).

\section{Conclusion}

Overall $10.6 \%$ of patients screened were ESBL-E carriers upon admission at HUG, mostly due to ESBL-producing E. coli. Patients admitted to septic orthopedics, geriatrics and ECS had a higher prevalence on admission. The majority of ESBL- Klebsiella pneumonia carriers had a recent history of hospitalization.

\section{Disclosure of interest}

None declared.

\section{Authors' details}

${ }^{1}$ Infection Control Programme, Geneva University Hospitals, Geneva, Switzerland. 'Bacteriology Laboratory, Geneva University Hospitals, Geneva, Switzerland. ${ }^{3}$ Geriatrics, Internal Medicine and Rehabilitation Service, Geneva University Hospitals, Geneva, Switzerland. ${ }^{4}$ Visceral Surgery Service, Geneva University Hospitals, Geneva, Switzerland. ${ }^{5}$ Anesthesiology Service, Geneva University Hospitals, Geneva, Switzerland. ${ }^{6}$ R-Gnosis WP5 Leader, CharitéUniversity Hospital, Berlin, Germany. ${ }^{7}$ R-Gnosis WP4 Leader, Sourasky Medical Center, Tel-Aviv, Israel.

Published: 16 June 2015

doi:10.1186/2047-2994-4-S1-P120

Cite this article as: Fankhauser et al.: Prevalence of extended-spectrum betalactamase producing-Enterobacteriaceae (ESBL-E) carriage on admission at Geneva University Hospitals (HUG). Antimicrobial Resistance and Infection Control 2015 4(Suppl 1):P120. 